International Journal of Pure and Applied Mathematics

Volume 85 No. 3 2013, 477-485

ISSN: 1311-8080 (printed version); ISSN: 1314-3395 (on-line version)

url: http://www.ijpam.eu

doi: http://dx.doi.org/10.12732/ijpam.v85i3.4

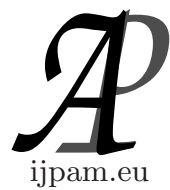

\title{
P-REGULAR NEARRINGS CHARACTERIZED BY THEIR BI-IDEALS
}

\author{
Aphisit Muangma $^{1}$, Aiyared Iampan ${ }^{2} \S$ \\ ${ }^{1,2}$ Department of Mathematics \\ School of Science \\ University of Phayao \\ Phayao, 56000, THAILAND
}

\begin{abstract}
Using the idea of quasi-ideals of $P$-regular nearrings, the concept of bi-ideals of $P$-regular nearrings is generalized, which is an extension of the concept of quasi-ideals of $P$-regular nearrings and some interesting characterizations of bi-ideals are obtained. As a result, we prove that every element of a bi-ideal $B$ of a $P$-regular nearring can be represented as the sum of two elements of $P$ and $Q$. Moreover, every element of the finite intersection $\bigcap_{i=1}^{n} B_{i}$ of bi-ideals of a $P$-regular distributive nearring $N$ can be represented as the
\end{abstract} sum of two elements of $P$ and $B_{1} N B_{2} N \ldots N B_{n-1} N B_{n}$.

AMS Subject Classification: 16Y30, 16D25

Key Words: $P$-regular nearring, quasi-ideal, bi-ideal

\section{Introduction and Preliminaries}

The notion of nearrings is first defined by Pilz [8] in 1977 and that of bi-ideals by Chelvam and Ganesan [3] in 1987. As we know, nearrings are a generalization of rings, and bi-ideals are a generalization of quasi-ideals and ideals in nearrings. Many types of ideals on the algebraic structures were characterized by several

Received: December 21, 2012

(C) 2013 Academic Publications, Ltd.

$\S_{\text {Correspondence author }}$ url: www.acadpubl.eu 
authors such as: In 1983, Yakabe [10] introduced and characterized the notion of quasi-ideals of nearrings. In 1987, Chelvam and Ganesan [3] introduced and generalized the notion of quasi-ideals of nearrings which was introduced by [10] to bi-ideals. In 1989, Yakabe [11] characterized regular zero-symmetric nearrings without nonzero nilpotent elements in terms of quasi-ideals. In 1990, Andrunakievich [2] introduced $P$-regular rings. In 1991, Choi [4] extended the $P$-regularity of rings which was introduced by [2] to the $P$-regularity of nearrings. In 2005, Kim, Jun and Yon [7] introduced the notion of anti fuzzy ideals of near-rings and investigated some related properties. In 2008, Abbasi and Rizvi [1] studied prime ideals in near-rings. In 2009, Zhan and B. Davvaz [12] introduced the concept of $(\bar{\epsilon}, \bar{\in} \vee \bar{q})$-fuzzy subnear-rings (ideals) of nearrings and obtain some of its related properties. In 2010, Choi [5] gave some characterizations of quasi-ideals of $P$-regular nearrings and proved that every element of a quasi-ideal $Q$ of a $P$-regular nearring can be represented as the sum of two elements of $P$ and $Q$. In 2011, Dheena and Manivasan [6] gave some characterizations of quasi-ideals of $P$-regular nearrings in the same way as of Choi [5]. In 2012, Sharma [9] studied the properties of intuitionistic fuzzy ideals of near ring with the help of their $(\alpha, \beta)$-cut sets. The concept of quasiideals play an important role in studying the structure of nearrings. Now, the notion of bi-ideals is an important and useful generalization of quasi-ideals of nearrings. Therefore, we will study bi-ideals of nearrings in the same way as of quasi-ideals of nearrings which was studied by Choi [5].

To present the main results we discuss some elementary definitions that we use later.

Definition 1.1. [8] A nearring is a system consisting of a nonempty set $N$ together with two binary operations on $N$ called addition and multiplication such that

(1) $N$ together with addition is a group,

(2) $N$ together with multiplication is a semigroup, and

(3) $(a+b) c=a c+b c$ for all $a, b, c \in N$.

For two nonempty subsets $A$ and $B$ of a nearring $N$, let

$$
A+B:=\{a+b \mid a \in A \text { and } b \in B\}
$$

and

$$
A B:=\{a b \mid a \in A \text { and } b \in B\} .
$$


If $A=\{a\}$, then we also write $\{a\}+B$ as $a+B$, and $\{a\} B$ as $a B$, and similarly if $B=\{b\}$.

Definition 1.2. A nonempty subset $S$ of a nearring $N$ is called a left (right) $N$-subgroup of $N$ if

(1) $S$ together with addition is a subgroup of $N$, and

(2) $N S \subseteq S(S N \subseteq S)$.

Definition 1.3. A nonempty subset $S$ of a nearring $N$ is called an ideal of $N$ if

(1) $S$ together with addition is a normal subgroup of $N$,

(2) $S N \subseteq S$,

(3) $N S \subseteq S$, and

(4) $n_{1}\left(n_{2}+s\right)-n_{1} n_{2} \in S$ for all $s \in S$ and $n_{1}, n_{2} \in N$.

Note that $S$ is a left ideal of $N$ if $S$ satisfies (1), (3) and (4), and $S$ is a right ideal of $N$ if $S$ satisfies (1) and (2).

Remark 1.4. By Definition 1.3, we have that

(1) $S$ is a left ideal of $N$ if and only if $S$ is a normal left $N$-subgroup of $N$ and $n_{1}\left(n_{2}+s\right)-n_{1} n_{2} \in S$ for all $s \in S$ and $n_{1}, n_{2} \in N$.

(2) $S$ is a right ideal of $N$ if and only if $S$ is a normal right $N$-subgroup of $N$.

Definition 1.5. A nearring $N$ is called a distributive nearring if $a(b+c)=$ $a b+a c$ for all $a, b, c \in N$.

Definition 1.6. A nonempty subset $Q$ of a nearring $N$ is called a quasiideal of $N$ if

(1) $Q$ together with addition is a subgroup of $N$, and

(2) $Q N \cap N Q \subseteq Q$.

Definition 1.7. A nonempty subset $B$ of a nearring $N$ is called a bi-ideal of $N$ if

(1) $B$ together with addition is a subgroup of $N$, and 
(2) $B N B \subseteq B$

Definition 1.8. A nearring $N$ is called regular nearring if for each $x \in N$ there exists $y \in N$ such that $x y x=x$.

Definition 1.9. Let $N$ be a nearring with unity and $P$ an ideal of $N$. Then $N$ is said to be $P$-regular nearring if for each $x \in N$ there exists $y \in N$ such that $x y x-x \in P$.

\section{Lemmas}

Before the characterizations of bi-ideals of nearrings for the main results, we give some auxiliary results which are necessary in what follows.

Lemma 2.1. [5] Let $N$ be a nearring and $P=\{0\}$. If $N$ is a $P$-regular nearring, then $N$ is a regular nearring.

Lemma 2.2. Let $\mathcal{B}$ be a nonempty family of bi-ideals of a nearring $N$. Then $\bigcap \mathcal{B}$ is a bi-ideal of $N$.

Proof. Clearly, $\bigcap \mathcal{B}$ together with addition is a subgroup of $N$. Now, for all $B \in \mathcal{B}$, we have

$$
\bigcap \mathcal{B N} \cap \mathcal{B} \subseteq B N B \subseteq B
$$

Thus $\bigcap \mathcal{B} N \cap \mathcal{B} \subseteq \bigcap \mathcal{B}$. Hence $\cap \mathcal{B}$ is a bi-ideal of $N$.

Corollary 2.3. Any finite intersection of bi-ideals of a nearring is a biideal.

Lemma 2.4. Every quasi-ideal of a nearring is a bi-ideal.

Proof. Let $Q$ be a quasi-ideal of a nearring $N$. Then $Q$ together with addition is a subgroup of $N$. Thus $Q N Q \subseteq Q N$ and $Q N Q \subseteq N Q$, so $Q N Q \subseteq$ $Q N \cap N Q \subseteq Q$. Hence $Q$ is a bi-ideal of $N$. 


\section{Main Results}

In this section, give some characterizations of bi-ideals of nearrings. Finally, we prove that every element of a bi-ideal $B$ of a $P$-regular nearring can be represented as the sum of two elements of $P$ and $Q$. Moreover, every element of the finite intersection $\bigcap_{i=1}^{n} B_{i}$ of bi-ideals of a $P$-regular distributive nearring $N$ can be represented as the sum of two elements of $P$ and $B_{1} N B_{2} N \ldots N B_{n-1} N B_{n}$.

Theorem 3.1. Let $N$ be a $P$-regular nearring. Then for each $n \in N$ there exists $n^{\prime} \in N$ such that $n^{\prime} n \in P$.

Theorem 3.2. Let $N$ be a $P$-regular distributive nearring. Then for every right ideal $R$ and every left ideal $L$ of $N$,

$$
(P+R) \cap(P+L)=P+R L .
$$

Theorem 3.3. Let $N$ be a $P$-regular nearring and $B$ a bi-ideal of $N$. Then every $x \in B$ there exist $p^{\prime} \in P$ and $b^{\prime} \in B$ such that $x=p^{\prime}+b^{\prime}$.

Proof. Let $x \in B$. Since $N$ is a $P$-regular nearring and $x \in B \subseteq N$, there exists $y \in N$ such that $x y x-x=p$ for some $p \in P$. Thus $x=-p+x y x$. Since $B$ is a bi-ideal of $N$, we have $x y x \in B N B \subseteq B$. Since $p \in P$ and $P$ together with addition is a subgroup of $N$, we have $-p \in P$. Put $p^{\prime}=-p$ and $b^{\prime}=x y x$. Thus

$$
x=-p+x y x=p^{\prime}+b^{\prime} \in P+B .
$$

Theorem 3.4. Let $N$ be a $P$-regular distributive nearring and $B_{1}$ and $B_{2}$ bi-ideals of $N$. If $b \in B_{1} \cap B_{2}$ and $x \in N$, then the element $b$ can be represented as

$$
b=p+b_{1} x_{1} b_{2} \text { and } b_{1} x_{1} b_{2} x P \subseteq P
$$

for some $p \in P, x_{1} \in N, b_{1} \in B_{1}$ and $b_{2} \in B_{2}$.

Proof. Let $b \in B_{1} \cap B_{2}$. Since $N$ is a $P$-regular nearring, there exists $x_{1} \in N$ such that $b x_{1} b-b \in P$. By Lemma 2.2, we have $B_{1} \cap B_{2}$ is a bi-ideal of $N$. Since $b \in B_{1} \cap B_{2}$, we have $b \in B_{1}$ and $b \in B_{2}$. By Theorem 3.3, we have $b=p_{1}+b_{1}$ for some $p_{1} \in P$ and $b_{1} \in B_{1}$, and $b=p_{2}+b_{2}$ for some $p_{2} \in P$ and $b_{2} \in B_{2}$. Since $b x_{1} b-b \in P$, we have $b x_{1} b-b=p_{3}$ for some $p_{3} \in P$. Thus $b=-p_{3}+b x_{1} b$. Hence

$$
b=-p_{3}+b x_{1} b
$$




$$
\begin{aligned}
& =-p_{3}+\left(p_{1}+b_{1}\right) x_{1}\left(p_{2}+b_{2}\right) \\
& =-p_{3}+p_{1} x_{1} p_{2}+p_{1} x_{1} b_{2}+b_{1} x_{1} p_{2}+b_{1} x_{1} b_{2} .
\end{aligned}
$$

Since $P$ is an ideal of $N$, we have $-p_{3}, p_{1} x_{1} p_{2}, p_{1} x_{1} b_{2}, b_{1} x_{1} p_{2} \in P$. Then $-p_{3}+$ $p_{1} x_{1} p_{2}+p_{1} x_{1} b_{2}+b_{1} x_{1} p_{2}=p_{4}$ for some $p_{4} \in P$. Thus $b=p_{4}+b_{1} x_{1} b_{2}$, so $b_{1} x_{1} b_{2}=-p_{4}+b$. Hence

$$
b_{1} x_{1} b_{2} x P=\left(-p_{4}+b\right) x P \subseteq-p_{4} x P+b x P \subseteq P+P \subseteq P .
$$

Theorem 3.5. Let $N$ be a $P$-regular distributive nearring and $\left\{B_{i} \mid i \in \mathbb{Z}\right.$ and $1 \leq i \leq n\}$ a nonempty family of bi-ideals of $N$. If $b \in \bigcap_{i=1}^{n} B_{i}$ and $x \in N$, then the element $b$ can be represented as

$$
b=p+b_{1} x_{1} b_{2} x_{2} \ldots b_{n-1} x_{n-1} b_{n} \text { and } b_{1} x_{1} b_{2} x_{2} \ldots b_{n-1} x_{n-1} b_{n} x P \subseteq P
$$

for some $p \in P, x_{1}, x_{2}, \ldots, x_{n-1} \in N$ and $b_{i} \in B_{i}$ for all $1 \leq i \leq n$.

Proof. If $b \in B_{1}$, then by Theorem 3.3, we have $b=p+b_{1}$ for some $p \in P$ and $b_{1} \in B_{1}$. Thus

$$
b_{1} x P=(-p+b) x P \subseteq-p x P+b x P \subseteq P+P \subseteq P .
$$

Assume that the theorem is true for integer $n-1$. Let $b \in \bigcap_{i=1}^{n} B_{i}$. Since $\bigcap_{i=1}^{n} B_{i} \subseteq$ $\bigcap_{i=1}^{n-1} B_{i}$ and $\bigcap_{i=1}^{n} B_{i} \subseteq B_{n}$, we have $b \in \bigcap_{i=1}^{n-1} B_{i}$ and $b \in B_{n}$. By assumption, we have

$$
b=p_{1}+b_{1} x_{1} b_{2} x_{2} \ldots b_{n-2} x_{n-2} b_{n-1}
$$

and $b_{1} x_{1} b_{2} x_{2} \ldots b_{n-2} x_{n-2} b_{n-1} x P \subseteq P$ for some $p_{1} \in P, x_{1}, x_{2}, \ldots, x_{n-2} \in N$ and $b_{i} \in B_{i}$ for all $1 \leq i \leq n-1$. By Theorem 3.3, we have

$$
b=p_{2}+b_{n}
$$

for some $p_{2} \in P$ and $b_{n} \in B_{n}$. Since $N$ is a $P$-regular nearring, there exists $x_{n-1} \in N$ such that $b x_{n-1} b-b \in P$. Thus $b x_{n-1} b-b=p_{3}$ for some $p_{3} \in P$, so $b=-p_{3}+b x_{n-1} b$. By (3.1) and (3.2), we have

$$
b x_{n-1} b=\left(p_{1}+b_{1} x_{1} b_{2} x_{2} \ldots b_{n-2} x_{n-2} b_{n-1}\right) x_{n-1}\left(p_{2}+b_{n}\right) .
$$

By (3.3), we have

$$
b=-p_{3}+b x_{n-1} b
$$




$$
\begin{aligned}
= & -p_{3}+\left(p_{1}+b_{1} x_{1} b_{2} x_{2} \ldots b_{n-2} x_{n-2} b_{n-1}\right) x_{n-1}\left(p_{2}+b_{n}\right) \\
= & -p_{3}+p_{1} x_{n-1} p_{2}+p_{1} x_{n-1} b_{n}+ \\
& b_{1} x_{1} b_{2} x_{2} \ldots b_{n-2} x_{n-2} b_{n-1} x_{n-1} p_{2}+ \\
& b_{1} x_{1} b_{2} x_{2} \ldots b_{n-2} x_{n-2} b_{n-1} x_{n-1} b_{n} .
\end{aligned}
$$

Put $-p_{3}+p_{1} x_{n-1} p_{2}+p_{1} x_{n-1} b_{n}+b_{1} x_{1} b_{2} x_{2} \ldots b_{n-2} x_{n-2} b_{n-1} x_{n-1} p_{2}=p_{4}$ for some $p_{4} \in P$. Thus

$$
b=p_{4}+b_{1} x_{1} b_{2} x_{2} \ldots b_{n-2} x_{n-2} b_{n-1} x_{n-1} b_{n} .
$$

That is $b_{1} x_{1} b_{2} x_{2} \ldots b_{n-2} x_{n-2} b_{n-1} x_{n-1} b_{n}=-p_{4}+b$. Hence

$$
\begin{aligned}
b_{1} x_{1} b_{2} x_{2} \ldots b_{n-2} x_{n-2} b_{n-1} x_{n-1} b_{n} x P & =\left(-p_{4}+b\right) x P \\
& \subseteq-p_{4} x P+b x P \\
& \subseteq P+P \subseteq P .
\end{aligned}
$$

Theorem 3.6. Let $N$ be a $P$-regular nearring and $B$ a bi-ideal of $N$. Then

$$
P+B=P+B N B \text {. }
$$

Proof. Since $B$ is a bi-ideal of $N$, we have $B N B \subseteq B$. Thus

$$
P+B N B \subseteq P+B .
$$

On the other hand, let $n \in P+B$. Then $n=p^{\prime}+b^{\prime}$ for some $p^{\prime} \in P$ and $b^{\prime} \in B$. Since $N$ is a $P$-regular nearring, there exists $x \in N$ such that $b^{\prime} x b^{\prime}-b^{\prime} \in P$. Thus $b^{\prime} x b^{\prime}-b^{\prime}=p^{\prime \prime}$ for some $p^{\prime \prime} \in P$, so $b^{\prime}=-p^{\prime \prime}+b^{\prime} x b^{\prime}$. Therefore

$$
n=p^{\prime}+b^{\prime}=p^{\prime}+\left(-p^{\prime \prime}+b^{\prime} x b^{\prime}\right)=\left(p^{\prime}-p^{\prime \prime}\right)+b^{\prime} x b^{\prime} \in P+B N B .
$$

Hence

$$
P+B \subseteq P+B N B .
$$

By (3.4) and (3.5), we have $P+B=P+B N B$.

Theorem 3.7. Let $N$ be a $P$-regular nearring, and $B_{1}$ and $B_{2}$ bi-ideals of $N$. Then

$$
P+\left(B_{1} \cap B_{2}\right) \subseteq P+\left(B_{1} N B_{2} \cap B_{2} N B_{1}\right) .
$$

Proof. Let $b \in P+\left(B_{1} \cap B_{2}\right)$. Then $b=p+b^{\prime}$ for some $p \in P$ and $b^{\prime} \in B_{1} \cap B_{2}$. Thus $b^{\prime} \in B_{1}$ and $b^{\prime} \in B_{2}$. Since $N$ is a $P$-regular nearring, there exists $x \in N$ such that $b^{\prime} x b^{\prime}-b^{\prime} \in P$. Thus $b^{\prime} x b^{\prime}-b^{\prime}=p^{\prime}$ for some $p^{\prime} \in P$, so $b^{\prime}=-p^{\prime}+b^{\prime} x b^{\prime}$. Hence 


$$
b=p+b^{\prime}=p-p^{\prime}+b^{\prime} x b^{\prime}=p^{\prime \prime}+b^{\prime} x b^{\prime} \in P+\left(B_{1} N B_{2} \cap B_{2} N B_{1}\right)
$$

where $p^{\prime \prime}=p-p^{\prime}$. Therefore

$$
P+\left(B_{1} \cap B_{2}\right) \subseteq P+\left(B_{1} N B_{2} \cap B_{2} N B_{1}\right) .
$$

Theorem 3.8. Let $N$ be a P-regular nearring, and $\left\{B_{i} \mid i \in \mathbb{Z}\right.$ and $1 \leq i \leq n\}$ a nonempty family of bi-ideals of $N$. Then

$$
\begin{gathered}
P+\left(\bigcap_{i=1}^{n} B_{i}\right) \subseteq \\
P+\left(B_{1} N B_{n} \cap B_{2} N B_{n} \cap \ldots \cap B_{n-1} N B_{n} \cap B_{n} N B_{1} \cap B_{n} N B_{2} \cap \ldots \cap B_{n} N B_{n-1}\right) .
\end{gathered}
$$

Proof. By Theorem 3.6, we have $P+B_{1}=P+B_{1} N B_{1}$. That is $P+B_{1} \subseteq$ $P+B_{1} N B_{1}$. Assume that the theorem is true for integer $n-1$. By Theorem 3.7, we have

$$
\begin{aligned}
P+\left(\bigcap_{i=1}^{n} B_{i}\right)= & P+\left(\bigcap_{i=1}^{n-1} B_{i} \cap B_{n}\right) \\
\subseteq & P+\left(\left(\bigcap_{i=1}^{n-1} B_{i}\right) N B_{n} \cap B_{n} N\left(\bigcap_{i=1}^{n-1} B_{i}\right)\right) \\
\subseteq & P+\left(\left(B_{1} \cap B_{2} \cap \ldots \cap B_{n-1}\right) N B_{n}\right. \\
& \left.\cap B_{n} N\left(B_{1} \cap B_{2} \cap \ldots \cap B_{n-1}\right)\right) \\
\subseteq & P+\left(B_{1} N B_{n} \cap B_{2} N B_{n} \cap \ldots \cap B_{n-1} N B_{n}\right. \\
& \left.\cap B_{n} N B_{1} \cap B_{n} N B_{2} \cap \ldots \cap B_{n} N B_{n-1}\right) .
\end{aligned}
$$

\section{Acknowledgments}

This research is supported by the Group for Young Algebraists in University of Phayao (GYA), Thailand.

The authors wish to express their sincere thanks to the referees for the valuable suggestions which lead to an improvement of this paper. 


\section{References}

[1] S. J. Abbasi and A. Z. Rizvi, Study of prime ideals in near-ring, Journal of Engineering and Sciences, 2 (2008), 65-66.

[2] V. A. Andrunakievich, Regularity of a ring with a respect to right ideals, Doklady Akademii Nauk SSSR, 310 (1990), 267-272.

[3] T. T. Chelvam and N. Ganesan, On bi-ideals of near-rings, Indian Journal of Pure and Applied Mathematics, 18 (1987), 1002-1005.

[4] S. J. Choi, P-regularity of a near-ring, Master's thesis, University of DongA, 1991.

[5] S. J. Choi, Quasideal of a P-regular near-ring, International Journal of Algebra, 4 (2010), 501-506.

[6] P. Dheena and S. Manivasan, Quasiideals of a P-regular near-rings, International Journal of Algebra, 5 (2011), 1005-1010.

[7] K. H. Kim, Y. B. Jun, and Y. H. Yon, On anti fuzzy ideals in near-rings, Iranian Journal of Fuzzy Systems, 2 (2005), 71-80.

[8] G. Pilz, Near-rings, North-Holland Publishing Company, New York, 1977.

[9] P. K. Sharma, Intuitionistic fuzzy ideals of near rings, International Mathematical Forum, 7 (2012), 769-776.

[10] I. Yakabe, Quasi-ideals in near-rings, Mathematical reports College of General Education Kyushu University, 14 (1983), 41-46.

[11] I. Yakabe, Regular near-rings without non-zero nilpotent elements, Proceedings of the Japan Academy, Series A, 65 (1989), 176-179.

[12] J. M. Zhan and B. Davvaz, Generalized fuzzy ideals of near-rings, Applied Mathematics-A Journal of Chinese Universities, 24 (2009), 343-349. 
